\title{
CONTINUITY OF RANDOM DERIVATIONS
}

\author{
M. V. VELASCO AND A. R. VILLENA
}

(Communicated by Palle E. T. Jorgensen)

\begin{abstract}
In this paper we extend the well-known Johnson-Sinclair theorem in a stochastic sense showing that stochastically derivative linear random operators on semisimple Banach algebras are stochastically continuous. Besides, we prove that probably derivative linear random operators on semisimple Banach algebras are probably continuous.
\end{abstract}

\section{INTRODUCTION}

A classical topic in the automatic continuity problem of linear operators is the problem of the automatic continuity of derivations. Many authors have been interested in this topic (see, e.g., $[2-4,6-9,11,15,16]$ ) since, twenty-five years ago, Johnson proved (in [10]) that derivations on complex commutative semisimple Banach algebras are continuous. For Banach algebras, the JohnsonSinclair theorem, which establishes that every derivation on a semisimple Banach algebra (real or complex) is continuous [11], is perhaps the most outstanding result in this line.

Our goal in this paper is to randomize the Johnson-Sinclair theorem. For that, we say that a linear random operator, $D$, on a Banach algebra $A$, is stochastically derivative when

$$
\mathbb{P}[D(a b)=D(a) b+a D(b)]=1, \quad \forall a, b \in A,
$$

and we show (Theorem 3.3.1) that stochastically derivative linear random operators, on semisimple Banach algebras, are stochastically continuous.

Now, following the pattern of [17], it is logical to weaken the condition of being stochastically derivative in the following way.

We say that a linear random operator, $D$, on a Banach algebra $A$ is probably derivative if there exists $0<\delta<1$ such that

$$
\mathbb{P}[D(a b)=a D(b)+D(a) b] \geq \delta, \quad \forall a, b \in A .
$$

In the last section of this paper our purpose is to inquire into the stochastic continuity of such operators.

For a probably derivative linear random operator, $D$, we define the number

$$
\gamma(D):=\inf \{\mathbb{P}[D(a b)=a D(b)+D(a) b]: a, b \in A\}
$$

Received by the editors April 5, 1993.

1991 Mathematics Subject Classification. Primary 46H40, 47B47, 47B80. 
(which in some sense can be considered as the probability of $D$ being stochastically derivative), and, in Corollary 4.3, we show that for a probably derivative linear random operator, $D$, on a semisimple Banach algebra, $A$, a measurable set, $\Omega_{0}$, with $\mathbb{P}\left[\Omega_{0}\right] \geq \gamma(D)$, can be got such that the linear random operator on $A, D_{/ \Omega_{0}}$, defined by $D_{/ \Omega_{0}}(a)=D(a)_{/ \Omega_{0}}$ being stochastically continuous.

\section{SOME TOPICS ABOUT THE CONTINUITY OF LINEAR RANDOM OPERATORS}

Throughout this paper, we consider a fixed probability space $(\Omega, \Sigma, \mathbb{P})$ and, given a Banach space, $Y$, we denote by $\mathscr{L}_{0}(\Omega, Y)$ the linear space of all $Y$ valued Bochner random variables on $(\Omega, \Sigma, \mathbb{P})$, which, with the almost surely (abbr. a.s.) identification, is an $F$-space with the convergence in probability topology [13].

A linear random operator from a Banach space $X$ to $\mathscr{L}_{0}(\Omega, Y)$ is called linear random operator from $X$ to $Y$.

It is said that a linear random operator, $T$, from $X$ to $Y$, is stochastically continuous when

$$
\left\{x_{n}\right\} \rightarrow x, \text { in } X \Rightarrow\left\{T\left(x_{n}\right)\right\} \rightarrow T(x), \text { in probability. }
$$

This property can be characterized as follows:

for every $0<\epsilon<1, \exists M>0$ such that $\mathbb{P}[\|T(x)\| \leq M\|x\|] \geq \epsilon, \quad \forall x \in X$.

Besides, if we denote the separating subspace for $T$ by $\mathscr{S}(T)$, i.e.,

$$
\mathscr{S}(T)=\left\{\mathbf{y} \in \mathscr{L}_{0}(\Omega, Y): \exists x_{n} \rightarrow 0 \text { with } T\left(x_{n}\right) \rightarrow \mathbf{y} \text { in probability }\right\},
$$

we have that, by the closed graph theorem,

$T$ is stochastically continuous $\Leftrightarrow \mathbb{P}[\mathbf{y}=0]=1, \quad \forall \mathbf{y} \in \mathscr{S}(T)$.

For a linear random operator, $T$, we define the real number

$$
\alpha(T):=\inf \{\mathbb{P}[\mathbf{y}=0]: \mathbf{y} \in \mathscr{S}(T)\} .
$$

Since $\alpha(T)$ is indeed a minimum (see [17]), it is clear that $T$ is stochastically continuous if and only if $\alpha(T)=1$.

Now, we consider linear random operators which behave as stochastically continuous ones on some measurable subset of $\Omega$. For that, let $\Omega_{0}$ be a measurable set, with $\mathbb{P}\left[\Omega_{0}\right]>0$, and let $T$ be a linear random operator from $X$ to $Y$. Considering $\Omega_{0}$ as a new probability space (with the inherited structure from $\Omega$ ), we can define the operator $T_{/ \Omega_{0}}: X \rightarrow \mathscr{L}_{0}(\Omega, Y)$ by $T_{/ \Omega_{0}}(x)=T(x)_{/ \Omega_{0}}$, and this linear random operator $T_{/ \Omega_{0}}$ is said to be a conditional operator of $T$.

We say that a linear random operator, $T$, is probably continuous when some of its conditional operators are stochastically continuous. Besides, if $T_{/ \Omega_{0}}$ is stochastically continuous then $\alpha(T) \geq \mathbb{P}\left[\Omega_{0}\right]$, trivially. Reciprocally, probably continuous linear random operators are these which have stochastically small separating subspace. To be exact, as we proved in [17, Theorem 7], if $T$ is probably continuous then, there exists a measurable set, $\Omega_{0}$, with $\mathbb{P}\left[\Omega_{0}\right]=\alpha(T)$, such that $T_{/ \Omega_{0}}$ is stochastically continuous. Moreover, the set

\section{$\left\{\mathbb{P}\left[\Omega_{0}\right]: T_{/ \Omega_{0}}\right.$ is stochastically continuous $\}$}

has a maximum which coincides with $\alpha(T)$. (In consequence, $\alpha(T)$ can be considered, in this sense, as the probability of $T$ being stochastically continuous.) 
We conclude this section observing that by [17, Corollary 8], if $T$ is a nonstochastically continuous linear random operator and $\delta$ is in $] \alpha(T), 1[$ then, for every positive constant $M$, there exists $x_{M}$ in $X$, such that

$$
\mathbb{P}\left[\left\|T\left(x_{M}\right)\right\| \geq M\left\|x_{M}\right\|\right]>1-\delta,
$$

so, the next result follows.

Theorem 2.1. If $T$ is not stochastically continuous then, for every $\delta$ in $] 0,1-$ $\alpha(T)\left[\right.$ and every positive random variable $Z$, we can find an element, $x_{\delta, z}$ in $X$, which can be choosen with arbitrary small norm, satisfying that

$$
\mathbb{P}\left[\left\|T\left(x_{\delta}, z\right)\right\| \geq Z\right] \geq \delta .
$$

\section{STOCHASTIC CONTINUITY OF STOCHASTICALLY DERIVATIVE LINEAR RANDOM OPERATORS}

The following lemma is essential for the conclusion of the basic results of this paper. Given a set, $J$, we denote by $\mathscr{F}(J)$ the family of all finite subsets of $J$.

Lemma 3.1. Let $\left\{Q_{j}: j \in J\right\}$ be a family of closed sets in a Banach space $Y$. Then, for every $\mathbf{y}$ in $\mathscr{L}_{0}(\Omega, Y)$,

$$
\mathbb{P}\left[\mathbf{y} \in \bigcap_{j \in J} Q_{j}\right]=\inf _{F \in \mathscr{F}(J)}\left\{\mathbb{P}\left[\mathbf{y} \in \bigcap_{j \in F} Q_{j}\right]\right\} .
$$

Proof. Let $\mathbf{y}$ be in $\mathscr{L}_{0}(\Omega, Y)$. By [14, Theorem 1.1], there exists a separable Banach space, $L$, (depending on $\mathbf{y}$ ) in which $\mathbf{y}$ take its values almost surely, so

$$
\mathbb{P}\left[\mathbf{y} \in \bigcap_{j \in J} Q_{j}\right]=\mathbb{P}\left[\mathbf{y} \in\left(\bigcap_{j \in J} Q_{j}\right) \cap L\right] .
$$

But, by [12, Theorem 13.1.3], there exists a sequence $\left\{j_{n}\right\}_{n \in \mathbb{N}}$, in $J$, such that

$$
L \backslash \bigcap_{j \in J} Q_{j}=\bigcup_{j \in J}\left(L \backslash Q_{j}\right)=\bigcup_{n \in \mathbb{N}}\left(L \backslash Q_{j_{n}}\right)=L \backslash\left(\bigcap_{n \in \mathbb{N}} Q_{j_{n}}\right),
$$

and, therefore,

$$
\begin{aligned}
\mathbb{P}\left[\mathbf{y} \in \bigcap_{j \in J} Q_{j}\right] & =\mathbb{P}\left[\mathbf{y} \in\left(\bigcap_{j \in J} Q_{j}\right) \cap L\right]=\mathbb{P}\left[\mathbf{y} \in\left(\bigcap_{n \in \mathbb{N}} Q_{j_{n}}\right) \cap L\right] \\
& \left.=\mathbb{P}\left[\mathbf{y} \in \bigcap_{n \in \mathbb{N}} Q_{j_{n}}\right)\right]=\lim _{n \rightarrow \infty} \mathbb{P}\left[\mathbf{y} \in \bigcap_{k=1}^{n} Q_{j_{k}}\right] .
\end{aligned}
$$

Moreover,

$$
\mathbb{P}\left[\mathbf{y} \in \bigcap_{j \in J} Q_{j}\right] \leq \mathbb{P}\left[\mathbf{y} \in \bigcap_{j \in F} Q_{j}\right], \quad \text { for all } F \text { in } \mathscr{F}(J),
$$

so the result follows. 
Throughout this section let $D$ be a stochastically derivative linear random operator on a Banach algebra, $A$. Moreover, the family of all primitive ideals of $A$ is denoted by $\left\{P_{i}\right\}_{i \in I}$, and we define the sets

$$
I_{1}:=\left\{i \in I: P_{i} \text { has infinite codimension }\right\}
$$

and

$$
I_{2}:=\left\{i \in I: P_{i} \text { has finite codimension }\right\} .
$$

3.1. Working with the primitive ideals of infinite codimension. The following lemma is a refinement of [11, Lemma 2.1].

Lemma 3.1.1. Let $X$ be an infinite-dimensional Banach space such that there exists a strictly irreducible representation of $A$ on $X$. Let $\mathfrak{D}$ be the centralizer of $A$ on $X$. If the vectors $x_{0}, x_{1}, \ldots \in X$ are linearly independent over $\mathfrak{D}$ then, given $y$ in $X \backslash\{0\}$, there exists $a$ in $A$ satisfying that

$$
a x_{0}=0, \quad a x_{1}=y, \text { and } a x_{1}, a x_{2}, \ldots \text { are } \mathfrak{D} \text {-independent } .
$$

Proof. By the Jacobson density theorem [5, Theorem 24.10], there exists $b_{1}$ in $A$ such that

$$
b_{1} x_{0}=0 \text { and } b_{1} x_{1}=y .
$$

Since $X$ is infinite-dimensional over $\mathfrak{D}$, we can find a vector, $y_{2}$, in $X$, which is not a linear combination (over $\mathfrak{D}$ ) of the vectors $b_{1} x_{1}, b_{1} x_{2}$. Again by the Jacobson density theorem, there exists $c_{2}$ in $A$, such that

$$
c_{2} x_{1}=c_{2} x_{0}=0 \text { and } c_{2} x_{2}=y_{2} \text {, }
$$

so, multiplying $c_{2}$ by a convenient constant, we obtain $b_{2}$, in $A$, satisfying that

$$
\left\|b_{2}\right\| \leq \frac{1}{2}, \quad b_{2} x_{1}=b_{2} x_{0}=0,
$$

and

$b_{2} x_{2}$ is not a linear combination (over $\mathfrak{D}$ ) of $b_{1} x_{1}$, and $b_{1} x_{2}$.

An analogous argument shows that, for all $n \geq 2$, we can get an element, $b_{n}$, in $A$, such that

$$
\left\|b_{n}\right\| \leq \frac{1}{2^{n}}, \quad b_{n} x_{n-1}=\cdots=b_{n} x_{0}=0,
$$

and

$b_{n} x_{n}$ is not a linear combination (over $\mathfrak{D}$ ) of $\left(\sum_{i=1}^{n-1} b_{i}\right) x_{1}, \ldots,\left(\sum_{i=1}^{n-1} b_{i}\right) x_{n}$.

Thus, defining $a:=\sum_{n=1}^{\infty} b_{n}$ we have that $a x_{0}=0$ and $a x_{1}=y$, obviously. Moreover, if $m<n$,

$$
a x_{n}=b_{n} x_{n}+\left(\sum_{i=1}^{n-1} b_{i}\right) x_{n}, a x_{m}=\left(\sum_{i=1}^{n-1} b_{i}\right) x_{m} \text { are } \mathfrak{D} \text {-independents. }
$$

Let $X$ be a Banach $A$-module. Given $x$ in $X$, we denote by $D_{x}$ the linear random operator, from $A$ to $X$, defined by $D_{x}(a)=D(a) x$, for all $a$ in $A$, (where $(D(a) x)(\omega)=D(a)(\omega) x, \forall \omega \in \Omega)$. 
Lemma 3.1.2. If $X$ is an infinite dimensional irreducible Banach A-module then, for all $x$ in $X$, the application $D_{x}: a \rightarrow D(a) x$ is stochastically continuous.

Proof. We assume that $y$ in $X$ is such that $D_{y}$ is not stochastically continuous, and we shall deduce a contradiction from this assumption.

We denote the centralizer of $A$ on $X$ by $\mathfrak{D}$. Since $X$ is infinite dimensional over $\mathfrak{D}$, there exists a countable set $\left\{x_{0}, x_{1}, \ldots\right\}$ of $\mathfrak{D}$-independent vectors in $X$ such that $\left\|x_{n}\right\|=1$. Applying inductively the last lemma, we obtain a sequence $\left\{a_{n}\right\}_{n \in \mathbb{N}}$ in $A$ satisfying that, for every $n$ in $\mathbb{N}$,

$$
\begin{aligned}
a_{n} \cdots a_{1} x_{n-1} & =0, \\
a_{n} \cdots a_{1} x_{n} & =y,
\end{aligned}
$$

and

$$
\left\{a_{n} \cdots a_{1} x_{j}, j \geq n\right\} \text { is } \mathfrak{D} \text {-independent. }
$$

Now, we can assume that

$$
\left\|a_{n}\right\| \leq \frac{1}{2^{n}}, \quad \forall n \in \mathbb{N}
$$

changing (1.2) by

$$
a_{n} \cdots a_{1} x_{n}=\tau_{n} y
$$

where $\tau_{n}$ is a convenient positive constant.

Since $D_{y}$ is not stochastically continuous, by Theorem 2.1 , there exists $0<\delta<1$ such that we can obtain, inductively, a sequence, $\left\{b_{n}\right\}_{n \in \mathbb{N}}$, in $A$, satisfying that

$$
\begin{gathered}
\left\|b_{n+1}\right\| \leq\left\|b_{n}\right\| \leq 1, \quad \forall n \in \mathbb{N}, \\
\mathbb{P}\left[\left\|b_{n}\right\| \leq\left(1+\left\|D\left(a_{j} \cdots a_{1}\right)\right\|\right)^{-1}\right] \geq 1-\frac{1}{n}, \quad 1 \leq j \leq n,
\end{gathered}
$$

and

$$
\mathbb{P}\left[\left\|D\left(b_{n}\right) \tau_{n} y\right\| \geq n+\sum_{j=1}^{n-1}\left\|D\left(b_{j} a_{j} \ldots a_{1}\right)\right\|\right] \geq \delta, \quad \forall n \geq 2 .
$$

We consider the elements

$$
c=\sum_{j=1}^{\infty} b_{j} a_{j} \cdots a_{1}
$$

and

$$
c_{n}=b_{n+1}+\sum_{j=n+2}^{\infty} b_{j} a_{j} \cdots a_{n+2}
$$

(given by series which are absolutely convergent).

Since

$$
\begin{aligned}
D(c)= & \sum_{j=1}^{n-1} D\left(b_{j} a_{j} \cdots a_{1}\right)+D\left(b_{n}\right) a_{n} \cdots a_{1}+b_{n} D\left(a_{n} \cdots a_{1}\right) \\
& \left.+D\left(c_{n}\right) a_{n+1} \cdots a_{1}+c_{n} D\left(a_{n+1} \cdots a_{1}\right) \quad \text { (a.s. }\right)
\end{aligned}
$$


we have, by (1.1) and (1.4), that

$$
\begin{aligned}
D(c) x_{n}= & \sum_{j=1}^{n-1} D\left(b_{j} a_{j} \cdots a_{1}\right) x_{n}+D\left(b_{n}\right) \tau_{n} y \\
& +b_{n} D\left(a_{n} \cdots a_{1}\right) x_{n}+c_{n} D\left(a_{n+1} \cdots a_{1}\right) x_{n} \quad \text { (a.s.). }
\end{aligned}
$$

Therefore,

$$
\begin{aligned}
\left\|D(c) x_{n}\right\| \geq & \left\|D\left(b_{n}\right) \tau_{n} y\right\|-\sum_{j=1}^{n-1}\left\|D\left(b_{j} a_{j} \cdots a_{1}\right)\right\| \\
& \left.-\left\|b_{n}\right\|\left\|D\left(a_{n} \cdots a_{1}\right)\right\|-\left\|c_{n}\right\|\left\|D\left(a_{n+1} \cdots a_{1}\right)\right\| \quad \text { (a.s. }\right),
\end{aligned}
$$

so that

$\mathbb{P}[\|D(c)\| \geq n-3] \geq \mathbb{P}\left[\left\|D(c) x_{n}\right\| \geq n-3\right]$

$$
\geq \mathbb{P}\left[\left\|D\left(b_{n}\right) \tau_{n} y\right\| \geq n+\sum_{j=1}^{n-1}\left\|D\left(b_{j} a_{j} \cdots a_{1}\right)\right\|,\right.
$$

$$
\begin{gathered}
\left.\left\|b_{n}\right\|\left\|D\left(a_{n} \cdots a_{1}\right)\right\| \leq 1,\left\|c_{n}\right\|\left\|D\left(a_{n+1} \cdots a_{1}\right)\right\| \leq 2\right] \\
\geq \mathbb{P}\left[\left\|D\left(b_{n}\right) \tau_{n} y\right\| \geq n+\sum_{j=1}^{n-1}\left\|D\left(b_{j} a_{j} \cdots a_{1}\right)\right\|\right] \\
+\mathbb{P}\left[\left\|b_{n}\right\|\left\|D\left(a_{n} \cdots a_{1}\right)\right\| \leq 1\right]+\mathbb{P}\left[\left\|c_{n}\right\|\left\|D\left(a_{n+1} \cdots a_{1}\right)\right\| \leq 2\right]-2 .
\end{gathered}
$$

But, we observe that

$$
\mathbb{P}\left[\left\|b_{n}\right\|\left\|D\left(a_{n} \cdots a_{1}\right)\right\| \leq 1\right] \geq \mathbb{P}\left[\left\|b_{n}\right\| \leq\left(1+\left\|D\left(a_{n} \cdots a_{1}\right)\right\|\right)^{-1}\right],
$$

so, by (1.6),

$$
\mathbb{P}\left[\left\|b_{n}\right\|\left\|D\left(a_{n} \cdots a_{1}\right)\right\| \leq 1\right] \geq 1-\frac{1}{n}, \quad \forall n \in \mathbb{N} .
$$

On the other hand, by (1.3) and (1.5), we have that

$$
\left\|c_{n}\right\| \leq 2\left\|b_{n+1}\right\|
$$

so

$$
\mathbb{P}\left[\left\|c_{n}\right\|\left\|D\left(a_{n+1} \cdots a_{1}\right)\right\| \leq 2\right] \geq \mathbb{P}\left[\left\|b_{n+1}\right\|\left\|D\left(a_{n+1} \cdots a_{1}\right)\right\| \leq 1\right]
$$

and, by (1.9),

$$
\mathbb{P}\left[\left\|D\left(c_{n}\right)\right\|\left\|D\left(a_{n+1} \cdots a_{1}\right)\right\| \leq 2\right] \leq\left(1-\frac{1}{n+1}\right) \geq\left(1-\frac{1}{n}\right) .
$$

In consequence it follows from (1.8), applying (1.7), (1.9), and (1.10), that

$$
\mathbb{P}[\|D(c)\| \geq n-3] \geq \delta+\left(1-\frac{1}{n}\right)+\left(1-\frac{1}{n}\right)-2=\delta-\frac{2}{n} .
$$

This contradiction proves the result. 
Lemma 3.1.3. For all b in $\mathscr{S}(D)$ the following is satisfied:

$$
\mathbb{P}\left[\mathbf{b} \in \bigcap_{i \in I_{1}} P_{i}\right]=1 \text {. }
$$

Proof. Let $P$ be an infinite-codimensional primitive ideal of $A$, and let $X$ be the left $A$-module corresponding to an irreducible representation of $A$ whose kernel is $P$ (see [5, Proposition 24.12]). Since $X$ is an infinite-dimensional irreducible Banach $A$-module, the previous lemma shows that the operator $D_{x}: a \rightarrow D(a) x$ is stochastically continuous, for all $x$ in $X$. Thus, if $\mathbf{b}$ is in $\mathscr{S}(D)$, it is satisfied that

$$
\mathbb{P}[\mathbf{b} x=0]=1, \quad \forall x \in X
$$

(because bx $\in \mathscr{S}\left(D_{x}\right)$ ), so denoting

$$
\operatorname{ker} x:=\{a \in A: a x=0\},
$$

we have a family, $\{\operatorname{ker} x: x \in X\}$, of closed subsets of $A$, such that

$$
\mathbb{P}\left[\mathbf{b} \in \bigcap_{x \in F} \operatorname{ker} x\right]=1,
$$

for every finite subset, $F$, of $X$. Therefore, Lemma 3.1 proves that

$$
\mathbb{P}\left[\mathbf{b} \in \bigcap_{x \in X} \operatorname{ker} x\right]=1,
$$

that is,

$$
\mathbb{P}[\mathbf{b} \in P]=1 \text {. }
$$

Now, applying Lemma 3.1 to the family $\left\{P_{i}: i \in I_{1}\right\}$, the result follows.

\subsection{Working with the primitive ideals of finite codimension.}

Lemma 3.2.1. Let $\left\{Q_{n}\right\}_{n \in \mathbb{N}}$ be a sequence of ideals of $A$ such that:

(i) $Q_{n}$ has finite codimension, $\forall n \in \mathbb{N}$,

(ii) $A / Q_{n}$ has a unit element, $\forall n \in \mathbb{N}$,

(iii) $Q_{n}+Q_{m}=A$, for all $n \neq m$.

Then, there exists a sequence, $\left\{a_{n}\right\}_{n \in \mathbb{N}}$, in $A$ satisfying that, for every $k$ in $\mathbb{N}$ :

(i) $a_{k}+Q_{n}=0, n=1, \ldots, k-1$,

(ii) $a_{k}+Q_{n}$ is invertible, $\forall n \geq k$,

(iii) $\left\|a_{k}\right\| \leq 1$.

Proof. In a first step, we show that if $n$ is in $\mathbb{N}$, then $Q_{m}+\bigcap_{j=1}^{n} Q_{j}=A, \forall m>$ $n$.

In effect, let $n:=\max \left\{k \in\{1, \ldots, m-1\}: Q_{m}+\bigcap_{j=1}^{k} Q_{j}=A\right\}$. If $n<$ $m-1$, then, for every $a$ in $A$, we have that there exists $a_{1}$ in $Q_{m}$ and $b_{1}$ in $\bigcap_{j=1}^{n} Q_{j}$ such that $a=a_{1}+b_{1}$. Let $u$ be a modular unit of $Q_{m}$ (which exists because $A / Q_{m}$ has a unit element). Since $A=Q_{m}+Q_{n+1}$, we can find $a_{2}$ in $Q_{m}$ and $b_{2}$ in $Q_{n+1}$ such that $u=a_{2}+b_{2}$, so that $a u=\left(a_{1}\left(a_{2}+b_{2}\right)+b_{1} a_{2}\right)+b_{1} b_{2}$ lies in $Q_{m}+\bigcap_{j=1}^{n+1} Q_{j}$. Therefore, $a=(a-a u)+a u$ belongs to $Q_{m}+\bigcap_{j=1}^{n+1} Q_{j}$, which contradicts the definition of $n$. 
Now, proceeding as in the proof of [18, Lemma 2.2] from the first step, it follows that the homomorphism $a \rightarrow\left(a+Q_{1}, \ldots, a+Q_{n}\right)$, from $A$ to $\bigoplus_{j=1}^{n} A / Q_{j}$ is onto, for all $n$ in $\mathbb{N}$. In consequence, the same argument used in [18, Lemma 2.6] proves the result.

Lemma 3.2.2. Let b be in $\mathscr{S}(D)$. Then, for every $0<\epsilon<1$, there exists a finite subset, $F_{\epsilon}$, of $I_{2}$ such that

$$
\mathbb{P}\left[\mathbf{b} \in \bigcap_{i \in I_{2} \backslash F_{\epsilon}} P_{i}\right] \geq \epsilon .
$$

Proof. We assume to the contrary that there exists $0<\epsilon_{0}<1$ such that

$$
\mathbb{P}\left[\mathbf{b} \in \bigcap_{i \in I_{2} \backslash F} P_{i}\right]<\epsilon_{0}, \quad \text { for all finite subsets } F \text { of } I_{2} .
$$

Since $\mathbb{P}\left[\mathbf{b} \in \bigcap_{i \in I_{2}} P_{i}\right]<\epsilon_{0}$, Lemma 3.1 shows that there exists a finite subset, $F_{1}$, of $I_{2}$ such that

$$
\mathbb{P}\left[\mathbf{b} \in \bigcap_{i \in I_{2}} P_{i}\right] \leq \mathbb{P}\left[\mathbf{b} \in \bigcap_{i \in F_{1}} P_{i}\right]<\epsilon_{0} .
$$

Thus, we obtain inductively, applying Lemma 3.1, a sequence of finite subsets of $I_{2}$, denoted by $\left\{F_{n}\right\}_{n \in \mathbb{N}}$, satisfying that,

$$
\mathbb{P}\left[\mathbf{b} \in \bigcap_{I_{2} \backslash\left(F_{1} \cup \ldots \cup F_{n-1}\right)} P_{i}\right] \leq \mathbb{P}\left[\mathbf{b} \in \bigcap_{i \in F_{n}} P_{i}\right]<\epsilon_{0}
$$

and

$$
F_{n} \cap\left(F_{1} \cup \cdots \cup F_{n-1}\right)=\varnothing, \quad \forall n>1 .
$$

Now we define

$$
Q_{n}=\bigcap_{i \in F_{n}} P_{i}, \quad \forall n \in \mathbb{N} .
$$

Since $A / Q_{n}$ is isomorphic to $\bigoplus_{i \in F_{n}}\left(A / P_{i}\right)$ (see [11, Lemma 3.1]), the codimension of $Q_{n}$ is finite. On the other hand $A / P$ is a primitve Banach algebra, for every primitive ideal, $P$ [5, Proposition 26.9], so it is semisimple [5, Proposition 24.14]. If, in addition, the codimension of $P$ is finite, then $A / P$ has a unit element [1, Theorem 3.1]. Thus, we also deduce that $A / Q_{n}$ has a unit element.

Since $\left(P_{n}+P_{m}\right) / P_{n}$ and $\left(P_{n}+P_{m}\right) / P_{m}$ are two-sided ideals of the simple algebras $A / P_{n}$ and $A / P_{m}$, respectively, we have that $P_{n}+P_{m}=A, \forall n \neq m$. Therefore, Lemma 2.1 of [18] proves that if $m$ is not in $F_{n}$ then $P_{k}+Q_{m}=A$, $\forall k \in F_{n}$, and, applying the same lemma again, we deduce that

$$
Q_{n}+Q_{m}=A, \quad \forall n \neq m \text {. }
$$

We just show that Lemma 3.2.1 can be applied, so there exists a sequence $\left\{a_{n}\right\}_{n \in \mathrm{N}}$, in $A$ satisfying that

$$
\begin{array}{ll}
a_{k}+Q_{n}=0, & \forall n=1, \ldots, k-1, \\
a_{k}+Q_{n} \text { is invertible, } & \forall n \geq k,
\end{array}
$$


and

$$
\left\|a_{k}\right\| \leq 1 \text {. }
$$

Now, let $\delta$ be in $] 0,1-\epsilon_{0}$ [. Since, for every natural number, $n$, the linear random operator from $A$ to $A / Q_{n}$, given by $a \rightarrow D(a)+Q_{n}$, and denoted by $\Pi_{Q_{n}} \circ D$, satisfies that

$$
\alpha\left(\Pi_{Q_{n}} \circ D\right)=\inf \left\{\mathbb{P}[\mathbf{y}=0]: \mathbf{y} \in \mathscr{S}\left(\Pi_{Q_{n}} \circ D\right)\right\} \leq \mathbb{P}\left[\mathbf{b} \in Q_{n}\right]<\epsilon_{0},
$$

we can get, by Theorem 2.1 , inductively, a sequence $\left\{b_{n}\right\}_{n \in \mathbb{N}}$, in $A$ such that

$$
\begin{gathered}
\left\|b_{n}\right\| \leq \frac{1}{2^{n}}, \quad \forall n \in \mathbb{N}, \\
\mathbb{P}\left[\left\|b_{n}\right\| \leq\left(1+\sum_{j=1}^{n}\left\|D\left(a_{j}\right)\right\|\right)^{-1}\right] \geq 1-\frac{1}{n}, \quad \forall n \in \mathbb{N},
\end{gathered}
$$

and

$$
\mathbb{P}\left[\begin{array}{l}
\left.\left\|D\left(b_{n}\right)+Q_{n}\right\| \geq \prod_{j=1}^{n}\left\|\left(a_{j}+Q_{n}\right)^{-1}\right\|\left(n+\sum_{j=1}^{n-1}\left\|D\left(b_{j} a_{j} \cdots a_{1}\right)\right\|\right)\right] \\
>\delta, \quad \forall n \geq 2 .
\end{array}\right.
$$

We define

$$
c:=\sum_{j=1}^{\infty} b_{j} a_{j} \cdots a_{1}
$$

Then, by (2.1),

$$
\begin{aligned}
D(c)+Q_{n}= & \sum_{j=1}^{n-1}\left(D\left(b_{j} a_{j} \ldots a_{1}\right)+Q_{n}\right)+\left(D\left(b_{n}\right)+Q_{n}\right)\left(a_{n}+Q_{n}\right) \cdots\left(a_{1}+Q_{n}\right) \\
& +\left(b_{n}+Q_{n}\right)\left(D\left(a_{n}\right)+Q_{n}\right) \cdots\left(a_{1}+Q_{n}\right) \\
& +\cdots+\left(b_{n}+Q_{n}\right)\left(a_{n}+Q_{n}\right) \cdots\left(D\left(a_{1}\right)+Q_{n}\right) \\
& \left.+\left(b_{n+1}+Q_{n}\right)\left(D\left(a_{n+1}\right)+Q_{n}\right)\left(a_{n}+Q_{n}\right) \cdots\left(a_{1}+Q_{n}\right) \quad \text { (a.s. }\right),
\end{aligned}
$$

and, since

$$
\left\|D\left(b_{n}\right)+Q_{n}\right\| \leq\left\|\left(D\left(b_{n}\right)+Q_{n}\right)\left(a_{n}+Q_{n}\right) \cdots\left(a_{1}+Q_{n}\right)\right\| \prod_{j=1}^{n}\left\|\left(a_{j}+Q_{n}\right)^{-1}\right\|,
$$

we have that, applying (2.2),

$$
\begin{aligned}
\|D(c)\| \geq & \left\|D(c)+Q_{n}\right\| \\
\geq & \left\|D\left(b_{n}\right)+Q_{n}\right\| \prod_{j=1}^{n}\left\|\left(a_{j}+Q_{n}\right)^{-1}\right\|^{-1}-\sum_{j=1}^{n-1}\left\|D\left(b_{j} a_{j} \cdots a_{1}\right)\right\| \\
& \left.-\left\|b_{n}\right\| \sum_{j=1}^{n}\left\|D\left(a_{j}\right)\right\|-\left\|b_{n+1}\right\|\left\|D\left(a_{n+1}\right)\right\| \quad \text { (a.s. }\right),
\end{aligned}
$$


and thus

$\mathbb{P}[\|D(c)\| \geq n-2]$

$$
\begin{aligned}
& \geq \mathbb{P}\left[\left\|D(c)+Q_{n}\right\| \geq n-2\right] \\
& \geq \mathbb{P}\left[\left\|D\left(b_{n}\right)+Q_{n}\right\| \prod_{j=1}^{n}\left\|\left(a_{j}+Q_{n}\right)^{-1}\right\|^{-1}-\sum_{j=1}^{n-1}\left\|D\left(b_{j} a_{j} \cdots a_{1}\right)\right\| \geq n,\right.
\end{aligned}
$$

$$
\begin{aligned}
& \left.\left\|b_{n}\right\| \sum_{j=1}^{n}\left\|D\left(a_{j}\right)\right\| \leq 1,\left\|b_{n+1}\right\|\left\|D\left(a_{n+1}\right)\right\| \leq 1\right] \\
\geq & \mathbb{P}\left[\left\|D\left(b_{n}\right)+Q_{n}\right\| \geq \prod_{j=1}^{n}\left\|\left(a_{j}+Q_{n}\right)^{-1}\right\|\left(n+\sum_{j=1}^{n-1}\left\|D\left(b_{j} a_{j} \cdots a_{1}\right)\right\|\right)\right] \\
& +\mathbb{P}\left[\left\|b_{n}\right\| \sum_{j=1}^{n}\left\|D\left(a_{j}\right)\right\| \leq 1\right]+\mathbb{P}\left[\left\|b_{n+1}\right\|\left\|D\left(a_{n+1}\right)\right\| \leq 1\right]-2 .
\end{aligned}
$$

Since

$$
\mathbb{P}\left[\left\|b_{n}\right\| \sum_{j=1}^{n}\left\|D\left(a_{j}\right)\right\| \leq 1\right] \geq \mathbb{P}\left[\left\|b_{n}\right\| \leq\left(1+\sum_{j=1}^{n}\left\|D\left(a_{j}\right)\right\|\right)^{-1}\right],
$$

we have, by (2.3), that

$$
\mathbb{P}\left[\left\|b_{n}\right\| \sum_{j=1}^{n}\left\|D\left(a_{j}\right)\right\| \leq 1\right] \geq 1-\frac{1}{n} .
$$

We also observe that

$$
\mathbb{P}\left[\left\|b_{n+1}\right\|\left\|D\left(a_{n+1}\right)\right\| \leq 1\right] \geq \mathbb{P}\left[\left\|b_{n+1}\right\| \leq\left(1+\sum_{j=1}^{n+1}\left\|D\left(a_{j}\right)\right\|\right)^{-1}\right],
$$

so by (2.3),

$$
\mathbb{P}\left[\left\|b_{n+1}\right\|\left\|D\left(a_{n+1}\right)\right\| \leq 1\right] \geq 1-\frac{1}{n+1} \geq 1-\frac{1}{n} .
$$

Finally, applying (2.6) and (2.7), it follows from (2.5) that

$$
\mathbb{P}[\|D(c)\| \geq n-2] \geq \delta+2\left(1-\frac{1}{n}\right)-2=\delta-\frac{2}{n}, \quad \forall n \in \mathbb{N},
$$

which is impossible.

\subsection{The main result.}

Theorem 3.3.1. Stochastically derivative linear random operators, on semisimple Banach algebras, are stochastically continuous.

Proof. Let $D$ be such an operator, and let b be in $\mathscr{S}(D)$. Given $0<\epsilon<1$, by Lemma 3.2.2, there exists a finite set, $F_{\epsilon}$, of $I_{2}$, such that

$$
\mathbb{P}\left[\mathbf{b} \in \bigcap_{i \in I_{2} \backslash F_{\epsilon}} P_{i}\right] \geq \epsilon
$$


and, by Lemma 3.1.3,

$$
\mathbb{P}\left[\mathbf{b} \in \bigcap_{i \in I_{1}} P_{i}\right]=1,
$$

so, if $\left(I_{1} \cup I_{2}\right) \backslash F_{\epsilon}$ is denoted by $I_{0}$,

$$
\mathbb{P}\left[\mathbf{b} \in \bigcap_{i \in I_{0}} P_{i}\right] \geq \epsilon .
$$

Since $A$ is semisimple, the mapping $a \rightarrow \bigoplus_{i \in F_{\epsilon}}\left(a+P_{i}\right)$, from $\bigcap_{i \in I_{0}} P_{i}$ to $\bigoplus_{i \in F_{\epsilon}}\left(A / P_{i}\right)$ is injective [5, Proposition 24.14]. Therefore, $\bigcap_{i \in I_{0}} P_{i}$ is a (semisimple) finite-dimensional subalgebra of $A$, so by [1, Theorem 3.1.1], it has a unit element, which is denoted by $e$. Let $\left\{a_{n}\right\}_{n \in \mathbf{N}}$ be a sequence in $A$ converging to zero and such that $\left\{D\left(a_{n}\right)\right\}_{n \in \mathbf{N}}$ converges to $\mathbf{b}$, in probability. Then $\left\{D\left(e a_{n}\right)\right\}_{n \in \mathbb{N}}$ converges to $e \mathbf{b}$, in probability, so $e \mathbf{b}$ lies in the separating subspace for the restriction of $D$ to $\bigcap_{i \in I_{0}} P_{i}$, that is stochastically continuous because $\bigcap_{i \in I_{0}} P_{i}$ is finite dimensional. Therefore, $e \mathbf{b}=0$ (a.s.), so that,

$$
\mathbb{P}[\mathbf{b}=0]=\mathbb{P}\left[e \mathbf{b}=0, \mathbf{b} \in \bigcap_{i \in I_{0}} P_{i}\right]=\mathbb{P}\left[\mathbf{b} \in \bigcap_{i \in I_{0}} P_{i}\right] .
$$

Thus, by (3.1), $\mathbb{P}[\mathbf{b}=0] \geq \epsilon$. Since $\epsilon$ is arbitrary, this proves that $\mathbb{P}[\mathbf{b}=0]=1$ (i.e., $D$ is stochastically continuous).

\section{Probably CONTINUITy OF PROBABly DERIVATIVE LINEAR RANDOM OPERATORS}

Given a probably derivative linear random operator, $D$, with $\gamma(D)=\delta$, we know that, for every $a, b$ in $A$, there exists a measurable set, $\boldsymbol{\Omega}_{a, b}$, with $\mathbb{P}\left[\Omega_{a, b}\right] \geq \delta$, such that

$$
D(a b)=D(a) b+a D(b) \quad(\text { a.s. }) \text { on } \Omega_{a, b} .
$$

The difficulty of working with probably derivative linear random operators arises because we do not know the relationship between $\Omega_{a, b}$ and $\boldsymbol{\Omega}_{c, d}$, for $a, b, c, d$ in $A$. The main result of this section shows that a measurable set $\Omega_{0}$, with $\mathbb{P}\left[\Omega_{0}\right]=\gamma(D)$, can be got, such that

$$
D(a b)=D(a) b+a D(b) \quad \text { (a.s.) on } \Omega_{0}, \quad \forall a, b \in A \text {. }
$$

This result is a straightforward consequence of the following theorem.

Multilinear uniform randomization principle 4.1. Let $X_{1}, \ldots, X_{n}, Y$ be Banach spaces and $T$ a multilinear random operator, from $X_{1} \times \cdots \times X_{n}$ to $Y$.

(i) If there exists $0<\delta<1$ such that

$$
\mathbb{P}\left[T\left(x_{1}, \ldots, x_{n}\right)=0\right] \geq \delta, \quad \forall\left(x_{1}, \ldots, x_{n}\right) \in X_{1} \times \cdots \times X_{n},
$$

then there exists a measurable set $\Omega_{0}$, with $\mathbb{P}\left[\Omega_{0}\right] \geq \delta$, such that $T_{/ \Omega_{0}}$ $=0$ (a.s.).

(ii) The set

$\left\{\mathbb{P}\left[\Omega^{\prime}\right]: \Omega^{\prime}\right.$ is measurable and $T_{/ \Omega^{\prime}}=0$ (a.s.) $\}$ 
has a maximum, the set

$$
\left.\mathbb{P}\left[T\left(x_{1}, \ldots, x_{n}\right)=0\right]:\left(x_{1}, \ldots, x_{n}\right) \in X_{1} \times \cdots \times X_{n}\right\}
$$

has a minimum, and these values coincide.

Proof. For simplicity, we assume that $T$ is bilinear. Now, we consider the number

$$
\gamma:=\inf \left\{\mathbb{P}[T(x, y)=0]: x \in X_{1}, y \in X_{2}\right\},
$$

and we prove, by induction, that

$$
\begin{aligned}
\mathbb{P}\left[T\left(x_{1}, y_{1}\right)=\right. & \left.0, \ldots, T\left(x_{n}, y_{n}\right)=0\right] \geq \gamma, \\
& \forall x_{1}, \ldots, x_{n} \in X_{1}, \forall y_{1}, \ldots, y_{n} \in X_{2} .
\end{aligned}
$$

For $n=1$ the result is true. We assume that the previous equality is satisfied for a natural number, $n$. Then, if $x_{1}, \ldots, x_{n+1}$ are in $X_{1}$ and $y_{1}, \ldots, y_{n+1}$ are in $X_{2}$, we have that for $\lambda, \beta$ in $\mathbb{R}^{+}$,

$$
\begin{array}{r}
\gamma \leq \mathbb{P}\left[T\left(x_{1}, y_{1}\right)=0, \ldots, T\left(x_{n-1}, y_{n-1}\right)=0, T\left(x_{n}-\beta x_{n+1}, y_{n}-\lambda y_{n+1}\right)=0\right] \\
=\mathbb{P}\left[T\left(x_{1}, y_{1}\right)=0, \ldots, T\left(x_{n}-\beta x_{n+1}, y_{n}\right)=T\left(x_{n}-\beta x_{n+1}, y_{n+1}\right)=0\right] \\
+\mathbb{P}\left[T\left(x_{1}, y_{1}\right)=0, \ldots, T\left(x_{n}-\beta x_{n+1}, y_{n}\right)=\lambda T\left(x_{n}-\beta x_{n+1}, y_{n+1}\right),\right. \\
\left.T\left(x_{n}-\beta x_{n+1}, y_{n+1}\right) \neq 0\right],
\end{array}
$$

so, if $\lambda \rightarrow \infty$, we obtain that

$\gamma \leq \mathbb{P}\left[T\left(x_{1}, y_{1}\right)=0, \ldots, T\left(x_{n}-\beta x_{n+1}, y_{n}\right)=0, T\left(x_{n}-\beta x_{n+1}, y_{n+1}\right)=0\right]$.

But

$$
\begin{aligned}
\mathbb{P} & \left.T\left(x_{1}, y_{1}\right)=0, \ldots, T\left(x_{n}-\beta x_{n+1}, y_{n}\right)=0, T\left(x_{n}-\beta x_{n+1}, y_{n+1}\right)=0\right] \\
\leq & \mathbb{P}\left[T\left(x_{1}, y_{1}\right)=0, \ldots, T\left(x_{n}, y_{n}\right)=T\left(x_{n+1}, y_{n}\right)=0,\right. \\
& \left.\quad T\left(x_{n}, y_{n+1}\right)=T\left(x_{n+1}, y_{n+1}\right)=0\right] \\
& +\mathbb{P}\left[T\left(x_{1}, y_{1}\right)=0, \ldots, T\left(x_{n}, y_{n}\right)=\beta T\left(x_{n+1}, y_{n}\right), T\left(x_{n+1}, y_{n}\right) \neq 0\right] \\
& +\mathbb{P}\left[T\left(x_{1}, y_{1}\right)=0, \ldots, T\left(x_{n}, y_{n+1}\right)=\beta T\left(x_{n+1}, y_{n+1}\right), T\left(x_{n+1}, y_{n+1}\right)=0\right],
\end{aligned}
$$

so, if $\beta \rightarrow \infty$, we have that

$$
\begin{aligned}
& \gamma \leq \mathbb{P}\left[T\left(x_{1}, y_{1}\right)=0, \ldots, T\left(x_{n}, y_{n}\right)\right.=0, T\left(x_{n+1}, y_{n}\right)=0, \\
&\left.T\left(x_{n}, y_{n+1}\right)=0, T\left(x_{n+1}, y_{n+1}\right)=0\right],
\end{aligned}
$$

and, particularly,

$$
\gamma \leq \mathbb{P}\left[T\left(x_{1}, y_{1}\right)=0, \ldots, T\left(x_{n}, y_{n}\right)=0, T\left(x_{n+1}, y_{n+1}\right)=0\right] .
$$

Now, let $\left\{\left(u_{n}, v_{n}\right)\right\}$ be a sequence in $X_{1} \times X_{2}$ such that

$$
\gamma \leq \mathbb{P}\left[T\left(u_{n}, v_{n}\right)=0\right]<\gamma+\frac{1}{n} .
$$

We define the measurable set

$$
\Omega_{0}=\bigcap_{n=1}^{\infty}\left[T\left(u_{1}, v_{1}\right)=0, \ldots, T\left(u_{n}, v_{n}\right)=0\right],
$$

and we observe that, by (4.1),

$$
\gamma \leq \mathbb{P}\left[T\left(u_{1}, v_{1}\right)=0, \ldots, T\left(u_{n}, v_{n}\right)=0\right]
$$


and, by (4.2),

$$
\mathbb{P}\left[T\left(u_{1}, v_{1}\right)=0, \ldots, T\left(u_{n}, v_{n}\right)=0\right]<\gamma+\frac{1}{n},
$$

so

$$
\mathbb{P}\left[\Omega_{0}\right]=\lim _{n \rightarrow \infty} \mathbb{P}\left[T\left(u_{1}, v_{1}\right)=0, \ldots, T\left(u_{n}, v_{n}\right)=0\right]=\gamma \geq \delta .
$$

Finally, if the equality $T_{/ \Omega_{0}}(x, y)=0$ (a.s.) is not held, for some $(x, y)$ in $X_{1} \times X_{2}$, then

$$
\mathbb{P}\left[T(x, y)=0, \Omega_{0}\right]<\mathbb{P}\left[\Omega_{0}\right]=\gamma .
$$

But

$$
\begin{aligned}
\mathbb{P}\left[T(x, y)=0, \Omega_{0}\right] \\
\quad=\mathbb{P}\left[\bigcap_{n=1}^{\infty}\left[T(x, y)=0, T\left(u_{1}, v_{1}\right)=0, \ldots, T\left(u_{n}, v_{n}\right)=0\right]\right] \\
\quad=\lim _{n \rightarrow \infty} \mathbb{P}\left[T(x, y)=0, T\left(u_{1}, v_{1}\right)=0, \ldots, T\left(u_{n}, v_{n}\right)=0\right],
\end{aligned}
$$

so that, for some natural $n$,

$$
\mathbb{P}\left[T(x, y)=0, T\left(u_{1}, v_{1}\right)=0, \ldots, T\left(u_{n}, v_{n}\right)=0\right]<\gamma,
$$

which contradicts (4.1). Therefore,

$$
\inf \left\{\mathbb{P}[T(x, y)=0]: x \in X_{1}, y \in X_{2}\right\}=\sup \left\{\mathbb{P}\left[\Omega^{\prime}\right]: T_{/ \Omega^{\prime}}=0 \text { (a.s.) }\right\}=\mathbb{P}\left[\Omega_{0}\right],
$$

and the result is proved.

The multilinear uniform randomization principle, applied to the bilinear random operator $T(a, b):=D(a b)-D(a) b-a D(b)$, for a probably derivative linear random operator, $D$, proves that we can obtain a measurable set, $\Omega_{0}$, with $\mathbb{P}\left[\Omega_{0}\right]=\gamma(D)$, such that the conditional operator $D_{/ \Omega_{0}}$ is stochastically derivative. Therefore, by Theorem $3.3 .1, D_{/ \Omega_{0}}$ is stochastically continuous, and thus " the probability of $D$ being stochastically continuous" $(\alpha(D))$ is at least " the probability of $D$ being stochastically derivative" $(\gamma(D))$.

Corollary 4.2. Every probably derivative linear random operator, $D$, on a semisimple Banach algebra, is probably continuous. Moreover,

$$
\alpha(D) \geq \gamma(D) \text {. }
$$

We observe that, if in the above corollary $\gamma(D)=1$ (i.e., $D$ is stochastically derivative), then Theorem 3.3.1 is obtained.

\section{REFERENCES}

1. A. A. Albert, Structure of algebras, Amer. Math. Soc. Colloq. Publ., vol. 24, Amer. Math. Soc., Providence, RI, 1961.

2. W. G. Bade and P. C. Curtis Jr., Prime ideals and automatic continuity problems for Banach algebras, J. Funct. Anal. 29 (1978), 88-103.

3. __ Continuity of derivations of Banach algebras, J. Funct. Anal. 16 (1974), 372-387.

4. W. G. Bade and H. G. Dales, Continuity of derivations from radical convolution algebras, Studia Math. XCV (1989), 59-91.

5. F. F. Bonsall and J. Duncan, Complete normed algebras, Springer-Verlag, New York, 1973. 
6. M. Bresar, Jordan derivations on semiprime rings, Proc. Amer. Math. Soc. 104 (1988), 1003-1006.

7. J. Cusak, Jordan derivations on rings, Proc. Amer. Math. Soc. 53 (1975), 321-324.

8. _ Automatic continuity and topologically simple radical Banach algebras, J. London Math. Soc. 12 (1977), 493-500.

9. R. V. Garimella, Continuity of derivations on some semiprime Banach algebra, Proc. Amer. Math. Soc. 99 (1987), 289-292.

10. B. E. Johnson, Continuity of derivations on commutative algebras, Amer. J. Math. 91 (1969), $1-10$.

11. B. E. Johnson and A. M. Sinclair, Continuity of derivations and a problem of Kaplansky, Amer. J. Math. 90 (1968), 1067-1073.

12. K. Kuratowski, Introducción a la teoria de conjuntos y a la topologia, Traducción de $\mathbf{R}$. Rodriguez Vidal, Vicens-Vives, 1966.

13. M. Ledoux and M. Talagrand, Probability in Banach spaces, Springer-Verlag, New York, 1991.

14. J. Pettis, On integration in vectors spaces, Trans. Amer. Math. Soc. 44 (1938), 277-304.

15. J. R. Ringrose, Automatic continuity of derivations of operator algebras, J. London Math. Soc. 2 (1972), 432-438.

16. S. Sakai, On a conjecture of Kaplansky, Tôhoku Math. J. 12 (1960), 31-33.

17. M. V. Velasco and A. R. Villena, A random closed graph theorem, submitted.

18. A. R. Villena, Continuity of derivations on a complete normed alternative algebra, J. Inst. Math. Comput. Sci. Math. Ser. 3 (1990), 99-106.

Departamento de Análisis Matemático, Universidad de Granada, 18071 Granada, SPAIN

E-mail address: mvvelasco@ugr.es

E-mail address: avillena@ugr.es 